Rel: perit. bisl. $7(2): 211-216 \quad(200)())$

(1) Faculad de Ciencias Biológicas UNMSM

\title{
La importancia del manejo comunal para la conservación de la fauna silvestre en las áreas naturales protegidas del Nororiente Peruano
}

\section{Importance of community management for the conservation of wildife in the protected natural areas in Northeastern Peru}

\author{
Pablo Puertas ${ }^{1}$, Richard Bodmer ${ }^{2}$, Arsenio Calle ${ }^{1}$ y Javier del Aquila ${ }^{1}$
}

\section{INTRODUCCIÓN}

La extracción de recursos naturales es una de las principales actividades de los habitantes amazónicos que viven en los bosques inundables y en tierra firme (Beckerman, 1994; Bodmer; 1994). Una de esas áreas se encuentra situada en el Talnuayo-Blanco, en la Reserva Comunal Tamshiyacu-Tahuayo (RCTT), y la otra en la zona del Canal del Puinahua y zona baja del Samiria-Marañón, en la Reserva Nacional Pacaya-Samiria (RNPS) (Fig. I). La población de esas áreas, como la de otros bosques tropicales, preferentemente explotan animales de tamaño mediano a grande, los cuales proveen de fuente proteica y contribuyen a su economía a través de la venta de carne de monte (Bodmer el al., 1997).

La fauna silvestre en los bosques tropicales es fácilmente sobreexplotada y las especies pueden ser rápidamente reducidas hasta la extinción (Robinson y Redford, 1994: Robinson y Bodmer, 1999). En consecuencia, tanto la sobrecaza como la sobreexplotación de otros recursos producen cambios en la dinámica poblacional de las especies y de hecho influyen en el desenvolvimiento de las comunidades humanas.

Algunas comunidades de la RCTT como de la RNPS han empezado a manejar la fau-

1 Programa Integral de Desarrollo y Conservación Pacaya Samiria Junglevagt for Amazonas (AIF-WWF/DK). Pevas 120, lquitos. Peru. E-mail: primales amonles.com

2 Universily of Kenl, al Canterbury. Canlerbury, KenlCT2 7NS, England. E-mail P.Bodmeroskc.ac.uk na silvestre como una alternativa de conservación (Bodmer y Puertas, 2000). Sin embargo, la implementación del manejo comunal de fauna silvestre requiere contar con datos y una metodología de campo que concuerde con los cleseos y realidades de la población local.

Consideramos que las sugerencias proporcionadas en este trabajo ayudarán a las comunidades locales y a los planificadores de las áreas naturales protegidals a establecer una caza más sostenible a través de la implementación de políticas de manejo comunal fundamentadas con información biológica relevante.

\section{Las poblaciones humanas en la RCTT y la RNPS}

La mayoria de habitantes rurales que habitan la RCTT son gente detribalizada conocidos como "riberenos" (Puertas y Bodmer 1993). Ellos comúnmente practican la pesca, agricultura, caza, extracción a pequeña escala de productos maderables y colección de otros producros del bosque como frutos, nueces y fibras (Coomes, 1992)

La población en la RNPS, denominada localmente como "ribereños", consiste en un 80\% de indigenas pertenecientes al grupo érnico Cocama-Cocamilla y cerca del $20 \%$ a otros grupos indígenas: mestizos y biancos (PPS 1995). 


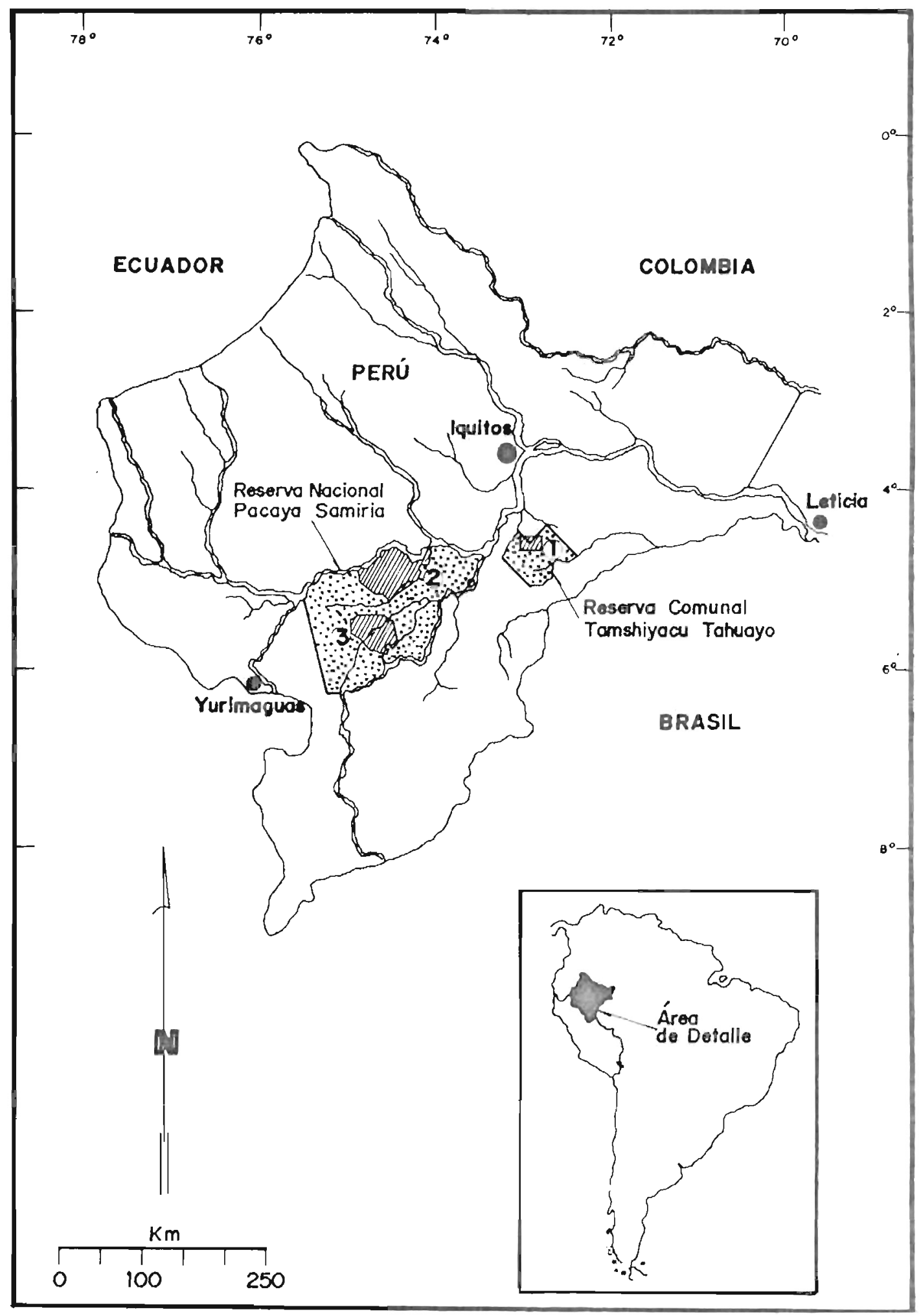

Fig. 1 Localización de las áreas de estudio en la Reserva Comunal Tamshiyacu-Tahuayo y Reserva Nacional Pacaya Samiria: 1) área del Tahuayo-Blanco, 2) área del Samiria-Marañón, y 3) área del Canal de Puinahua. 


\section{La metodología participativa para el ma- nejo de fauna silvestre}

En este trabajo destacamos el método participativo utilizado con las comunidades locales de la RCTT y RNPS para la elaboración y el desarrollo de planes de manejo de fauna silvestre.

El método participativo se basa en generar interés en las comunidades locales para el manejo de fauna silvestre involucrando a investigadores y extensionistas o promotores a trabajar con los cazadores cuando se evalúa el impacto de las cosechas (Bodmer y Puertas, 2000). Uno de esos métodos es el registro de la caza que incluye a la familia de los cazadores en la colección de datos. Este método participativo ayuda a los investigadores, promotores y cazadores a encontrar caminos comunes para discutir asuntos sobre fauna silvestre.

Observaciones directas fueron realizadas con la ayuda de cazadores locales que viven en la parte media del río Blanco. Tres cazadores con sus respectivas familias fueron capacitados durante 1993 en cómo registrar la actividad de caza; sin embargo, sólo dos de ellos colaboraron de manera efectiva. La vivienda de estos cazadores llamados localmente inspectores o registradores de caza se encontraban estratégicamente ubicados a la orilla del río Blanco a fin de visualizar y registrar con facilidad a los cazadores que iban ya sea de subida o de bajada. La colaboración de la mujer en los registros de caza fue muy valiosa. Ellas continuaron registrando la actividad de caza cuando el marido iba de cacería o cuando llevaba a vender productos agropecuarios a la ciudad de lquitos.

Cuando algunos cazadores no fueron controlados oportunamente, lo fueron indirectamente a través de la información proporcionada por cazadores o informantes clave.

\section{Fortalecimiento del sistema de manejo comunal}

En la actualidad el manejo de fauna silvestre con participación comunitaria representa una verdadera alternativa de conservación. Sin embargo, requiere de un proceso de manejo comunaì a fin de asegurar en el largo plazo un manejo más efectivo.

El manejo comunal se basa en la adquisición y comunicación de la información. Para ello, se necesita utilizar el siguiente diseño: hacer que la población local implemente el manejo de las poblaciones de caza. Los estudios biológicos sobre la caza de animales proveen información sobre el impacto de la caza y su efectividad de manejo. Simultáneamente, la investigación y la promoción agroforestal con énfasis en recursos agroforestales alternativos proveen información sobre el mejoramiento de hábitats de fauna silvestre y su efectividad de manejo. Resultados de tales estudios son luego transmitidos a la gente local a través de actividades de extensión. Por consiguiente las investigaciones tienden el puente que liga las realidades de la biología poblacional hacia el manejo comunal de recursos naturales (Bodmer y Puertas 2000).

El manejo comunitario debe incluir a los siguientes actores: comunidades locales, promotores de Organismos No Gubernamentales (ONGs), funcionarios de Organismos Gubernamentales (OGs) e investigadores (Bodmer y Puertas, 2000). Asimismo, es necesario considerar a los actores secundarios como comerciantes, localmente denominados "regatones".

Un paso importante del manejo comunal de fauna silvestre fue iniciado a partir de noviembre de 1993 en la zona del Tahuayo-Blanco y centralizada en la Comunidad El Chino. Se realizaron acuerdos comunales para el cumplimiento de un reglamento de caza con la participación de moradores y autoridades políticas de las comunidades Esperanza, Bue- 
na Vista, El Chino y pobladores del río Blanco. A través de las reuniones comunales y explicando a cada morador los alcances del reglamento, se promovió la participación masiva de la población local. Posteriormente, el 06 de marzo de 1994 en asamblea general con participación de dichas comunidades y los principales actores dicho reglamento fue sustentado y aprobado. El reconocimiento legal a través de la firma del reglamento dando amparo a los acuerdos comunales fue dado el 27 de marzo du 1994.

En la actualidad, los habitantes del río Blanco coordinan las acciones de manejo comunal con los habitantes de las comunidades aledañas del río Tahuayo sobre el accionar de la reserva, así como también con los principales actores a nivel local. Sin embargo, se requiere una efectiva presencia e integración de los extensionistas de fauna con los lideres de las comunidades beneficiarias. También, se requiere du un constante flujo de información entre el investigador y el extensionista, a fin de que este último sea un buen receptor y transmita adecuadamente la información recibida a los miembros de las comunidades encargadas del manejo. Experiencias anteriores sugieren que una inadecuada transmisión de la información efectuada por los trabajadores de extensión puede hacer fracasar las acciones del manejo comunal (Bodmer y Puertas, 2000). Desde luego, es necesaria una constante capacitación de los trabajadores de extensión hecha por expertos en el manejo comunal de fauna silvestre. Por consiguiente, el principal propósito de este estudio es que en e] largo plazo las propias comunidades puedan manejar sus acividades de caza a través de la información obtenida por ellos mismos sobre el esfuerzo de caza.

Una vez fortalecido el sistema de manejo comunal a nivel local, el paso siguiente sería buscar el respaldo político a nivel regional. Ello para obtener el respaldo legal a los acuerdos y gestiones que las comunidades tiendan a rea- lizar en el sistema de manejo informal y empirico. Para el logro de esta labor, las comunidades tienen que tener el respaldo de los principales actores a nivel local. Por otro lado, deben estar ya sea debidamente organizadas - capacitadas para llevar a cabo el manejo comunal.

Importancia del manejo comunal para la conservación de las áreas protegidas

Una manera promisoria para manejar los animales de caza en las áreas protegidas como la RNPS y la RCTT sería tener áreas donde no se realice caza, o "áreas fuente" en la zona protegida a fin de reponer las áreas con caza persistente o "áreas sumidero" tanto en las áreas de uso como en las áreas de amortiguamiento. Asimismo, el manejo de los animales de caza debería tener una fuerte participación comunal. Los esfuerzos comunales solamente llevarían a una conservación exitosa si las realidades económicas de la gente local operan con los requerimientos biológicos de las especies. Indudablemente, la conservación comunal fracasará si no se toman en consideración ciertos atributos biológicos de las especies.

Tanto el uso sostenible como las estrategias fuente-sumidero deberían considerarse en las iniciativas de conservación comunal en las áreas naturales protegidas de la Amazonía con énfasis en la RNPS y la RCTT. Las comunidades locales de ambas áreas protegidas necesitan reconocer el valor de considerar las zonas sin caza como poblaciones fuente, ello a fin de garantizar el uso sostenible a largo plazo de sus recursos. Las comunidades que reconocen y consideran las áreas protegidas como zonas fuente lo hacen porque concuerda con la necesidad de usar la fauna silvestre de acuerdo a su realidad socioeconómica. Con las zonas fuente la caza será más sostenible y los esfuerzos del manejo comunal tendrán más posibilidad de ser exitosos. Es decir, las comunidades tendrán más interés en proteger las areas debido al beneficio que obtendrían de un sistema de caza más sostenible. Desde Jue- 
go, ello decrecería los conflictos entre los guardaparques y las comunidades locales y podría ser una alternativa financieramente sostenible para las áreas naturales protegidas.

\section{Alternativas para el manejo comunal de fauna silvestre}

Una alternativa viable para la conservación de los bosques amazónicos debe ser con la participación de las cumunidades locales. Tanto 1. R RNPS como Ja RCTT representan excelentes modelos para esa alternativa. Sin embargo, para alcanzar los objetivos a largo plazo, el sistema de manejo comunal requiere ser fortalecido, los hábitats de la fauna silvestre necesitan ser mejorados; la caza, pesca, recursos forestales y otros recursos naturales necesitan también ser manejados por la gente local. Para alcanzar esta última meta, es muy importante que la gente local y las autoridades de gobierno estén conscientes de la información sobre el recurso que se va a manejar.

La comunicación de la información necesita realizarse en un lenguaje con terminologías entendibles para promover su adecuado entendimiento y ampliar la participación en el análisis de uso del recurso que se va a manejar. En el futuro la gente local analizaría la situación de sus recursos por sí misma para luego decidir, mediante la implementación de un sistema participativo, el manejo de la fauna silvestre.

Se recomienda que la dirección para la conservación de la fauna silvestre tanto en la RNPS como en la RCTT tenga en consideración los siguientes criterios: I) el uso sostenible y las estrategias fuente-sumidero necesitan incluirse en las iniciativas de conservación comunal en las áreas naturales protegidas de la Amazonía, con énfasis en la RNPS y la RCTT; 2) buena actitud de las autoridades de gobierno, así como de los investigadores y trabajadores de extensión o promotores para proveer adecuada información técnica y respe- tar las realidades socioeconómicas de la gente local; 3) capacidad de una coordinación efectiva; 4) mucha energía y fuerza de voluntad por parte de las autoridades de gobierno para apoyar las gestiones del manejo comunal de fauna silvestre; 5) sentido común para entender tanto las necesidades de la gente local como las tendencias actuales de la cooperación técnica internacional: 6) desarrollo de alternativas económicas viables ante la extracción no sostenible de la fauna silvestre; y 7 ) conocimiento de la biología de las especies. Para lograr que en la RCTT y la RNPS los programas de manejo de fauna silvestre sean más sostenibles es necesario elaborar planes de manejo comprensivos que permitan cierta flexibilidad en las prácticas y generen un flujo de información según el aprendizaje individual. Eso dará lugar a la elaboración de programas adaptativos que con apoyo del componente de extensión recomienden a la gente local - ya sea cazadores o pescadoris u otros manejadores - ajustar el uso de los recursos naturales según los línites sostenibles. Elio, según las características biológicas de las especies y las posibilidades de comercialización.

\section{AGRADECIMIENTOS}

Agradecemos al Instituto Nacional de Recursos Naturales (INRENA) y al Ministerio de Agricultura del Perú por las autorizaciones concedidas, así como al Centro de Investigaciones Veterinarias y de Altura (IVITA) de la Universidad Nacional Mayor de San Marcos (UNMSM) y a la University of Florida. USA por las facilidadus concedidas. Este trabajo fue financiado por Wildlife Conservation Society, The Chicago Zuological Society, Junglevagt for Amazonas (AIF-WWF/DK), Rainforest Conservation Fund (RCF) y la Asociación para la Conservación de la Amazonia (ACA). Estamos en deuda por el invalorable apoyo proporcionado por las comunidades de la Reserva Nacional Pacaya Samiria y la Reserva Comunal Tamshiyacu-Tahuayo. 


\section{LITERATURA CITADA}

Beckerman, S. 1994. Hunting and fisning in Amazonia: Hold the Answers, What are the questions?, pp. 177-200, en A. Roosevelt (ed.): Amazonian Indians from Prehistory to the Present, Anthropological Perspectives, University of Arizona Press, Tucson.

Bodmer, R. 1994. Managing wildlife with local communities: The case of the Reserva Comunal TamshiyacuTahuayo, pp. 113-134, en D. Western, M. Wright y S. Strum (eds.): Natural Connections: Perspectives on Community-Based Management, Island Firess, Washington DC.

Bodmer, R., J. Penn, P. Puertas, L. Moya y T. Fang. 1997. Linking conservation and local people through sustainable use of natural resources: Community-based management in the Peruvian Amazon, pp. 315-358, en C. Freese (ed.): Harvesting Wild Species: Implications for Biodiversity Conservation, The John Hopkins University Press, Baltimore, MD.
Bodmer, R. y P. Puertas. 2000. Community Based Co-Management of Wildlife in the Peruvian Amazon, en: J. Robinson and L. Bennet (eds.): Hunting of Tropical Wildlife, University of Chicago Press.

Coomes, O. 1992. Making a Living in the Amazon Kainforest: Peasants, Land, and Economy in the Tahuayo River Basin of Northeastern Peru. Tesis de doctorado, University of Wisconsin-Madison.

Programa Integral de Desarrollo y Conservación Pacaya-Samiria (PPS). 1995. Propuesta de Proyecto Segunda Fase, 25 pp.

Puertas, P. y R. Bodmer. 1993. Conservation of a High Diversity Primate Assemblage. Biodiversity and Conservation, 2:586-593.

Robinson, J. G. y R. Bodmer. 1999. Invited paper: Towards wildlife management in tropical forests. Journal of Wildlife Management, $63(1): 1-13$.

Robinson, J. and K. Redford. 1994. Measuring the sustainability of hunting in tropical forests. Oryx, 25(4): 249-256. 